\title{
SEM Study of the Photocatalytic Activity of SnO2 Films Exposed to UV Radiation Against the Human Pathogen C. albicans
}

Carlos Arzate-Quintana ${ }^{1}$, Javier Camarillo-Cisneros ${ }^{1}$, Paula Rebeca Realyvazquez-Guevara ${ }^{2}$, Alejandro Faudoa-Arzate $^{2}$, and Hazel Jaynelle Morales Rodríguez ${ }^{3}$

${ }^{1}$ Facultad de Medicina y Ciencias Biológicas, Universidad Autónoma de Chihuahua, Chihuahua, Chihuahua, Mexico, ${ }^{2}$ Tecnológico de Monterrey, Chihuahua, Chihuahua, Mexico, ${ }^{3}$ Universidad Tecnológica sur, Chihuahua, Chihuahua, Mexico

Corresponding author: Email: carzate@uach.mx

\section{Introduction}

Antibiotic resistance has become an important worldwide health problem in a short period of time, and the design of new antibiotics is a process that requires years of research and a high amount of resources [1], not to mention that microbes will eventually acquire resistance to the new chemotherapies $[2,4]$. In an effort to help solving this situation is necessary to approach it using different strategies, including the prevention or minimization of disease contagion. Physical methods of sterilization should be taken in consideration since they are quick and cheap methods [3]. Additionally, living organisms have no meanings of developing resistance mechanisms against them in the short term it is likely that techniques based on physical methods will still be useful for a long period of time. UV light has been used for decades as a very efficient wide range antimicrobial method, and resistance mechanisms had not been an important factor that could affect disinfection performance. Certain metal oxides, like $\mathrm{SnO} 2$ have photocatalytic properties that allow them to increase the efficacy of UV light antimicrobial activity [5-7]. In the present work antibacterial testing was performed employing $\mathrm{SnO} 2$ substrate coupled with UV light to measure the ensemble activity against $C$. albicans a common pathogen in the hospital environment from the microbiologic and scanning electron microscopy perspective.

Thin films of $\mathrm{SnO} 2$ were synthesized by spray pyrolysis method using chloride solution. The solution was prepared by diluting $6 \mathrm{~g}$ of SnCl4.5H2O in a solution of $5 \mathrm{ml}$ of methanol and $0.5 \mathrm{ml}$ of $\mathrm{HCl}$ and heated at $60 \mathrm{oC}$ for 10 minutes, then a solution consisting in $2 \mathrm{ml}$ of distilled water and $5 \mathrm{ml}$ of methanol was added in stirring for 10 minutes, this solution was then diluted to $50 \mathrm{ml}$ in a volumetric flask. The solution was then deposited in clean glass microscope slides heated at 80oC until complete dryness. Thin film chemical composition and characterization was performed by SEM microscopy and X-ray diffraction. Crystalline characteristics were obtained by adjusting XRD by means of the Rietveld method and compared against bulk values obtained by means of Density Functional Theory (DFT). Inhibition activity of UV light was performed by adding $20 \mu \mathrm{l}$ of a solution containing 4 x 106 yeast $/ \mathrm{ml}$ in six glass covered by $\mathrm{SnO} 2$ thin films. The films were divided in two groups of three replicas, one used as control with no exposure to UV light and the second exposed to UV light. In order to test the photocatalytic microbial inhibition three replicas were exposed to UV radiation during 15 minutes. All slides were then analyzed by SEM microscopy to observe the presence of yeast cells in the surface of the $\mathrm{SnO} 2$ thin films. The inhibition percentage of yeast cells was measured using the Breed method consisting in observing the slides using a Gram stain procedure and counting the number of cells in an optical microscope. The inhibition percentage was determined by comparing the number of cells observed in thin films not exposed to UV light, cells exposed to UV in absence of $\mathrm{SnO} 2$ thin films and with the films containing cells that were exposed to UV light. 


\section{Results}

Figure 1a) shows scanning electron micrograph of $\mathrm{SnO} 2$ thin film deposited in a glass surface in a scale of $50 \mu \mathrm{m}$. The EDS elemental analysis of the film is shown in figure 1b), which corroborates the expected elements present in the film. From refined XRD were calculated lattice parameters of $a=b=4.832 \AA$ y $c=$ $3.215 \AA$ slightly higher than reported for bulk $(a=b=4.7331 \AA, c=3.181 \AA$, JCPDS 41-1445). After analysis by the Rietveld method, the adjusted pattern shows preferential orientation on the $\mathrm{C}$-axis in which the $\mathrm{SnO} 2$ crystals grow in smaller size. Because of this, most of the particles should be oriented with the family of planes $\left[\begin{array}{lll}0 & 0 & 1\end{array}\right]$. We attribute it to the structural relaxation that the crystalline structure has on the surface. This hypothesis agrees with the model calculated by means of DFT, where increasing the degrees of freedom in the plane $\left(\begin{array}{lll}1 & 0 & 0\end{array}\right)$ also allows to have higher values than the reported bulk $(\mathrm{a}=\mathrm{b}=4.8964 \AA \mathrm{y}$ $\mathrm{c}=3.2840 \AA$, JCPDS 41-1445). Figure $2 \mathrm{a}$-b shows yeast cells adhered to the surface of the $\mathrm{SnO} 2$ thin film before exposure to UV light. Figure 2a shows an amplification of 1,500X in which a high density of cells can be observed. In figure $2 \mathrm{~b}$, with an amplification of 7,500X, shows with more detail the structure of the yeast cells, and even though no structural damage is observed, a slight dehydration is evident.

The microbiologic studies demonstrated that yeast cells deposited in a glass surface exposed to 15 minutes of UV light $(254 \mathrm{~nm})$ reached a $34 \%$ inhibition growth, and yeast cells on a $\mathrm{SnO} 2$ thin film, exposed to the same amount of UV radiation reached a $66 \%$ growth inhibition, results that were expected according to $\mathrm{SnO} 2$ photocatalytic activity reported in the scientific literature [2,5,6]. A comparison between yeast cells deposited on $\mathrm{SnO} 2$ thin films, with and without exposure to UV light, is shown in Figure 2c-d. Cell density is higher in figure $2 \mathrm{c}$, which corresponds to the non treated film. A lower number of yeast cells are shown on figure $2 \mathrm{~d}$, that consist on the films exposed to 15 minutes of UV light.

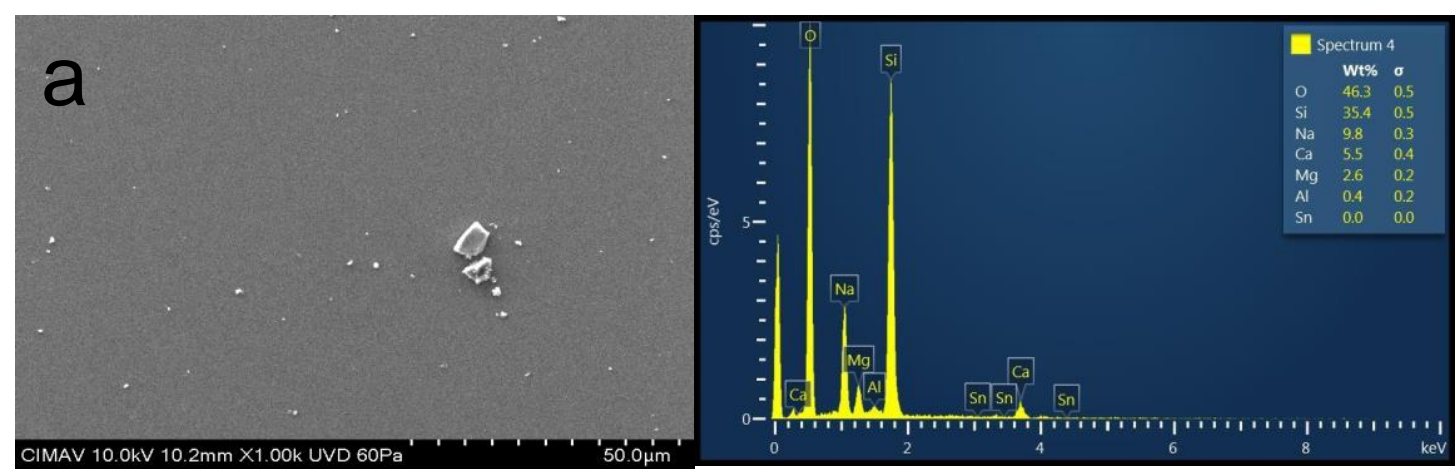

Figure 1. a) SEM Micrograph of $\mathrm{SnO}_{2}$ thin film, b) EDS analysis of $\mathrm{SnO}_{2}$ thin film 

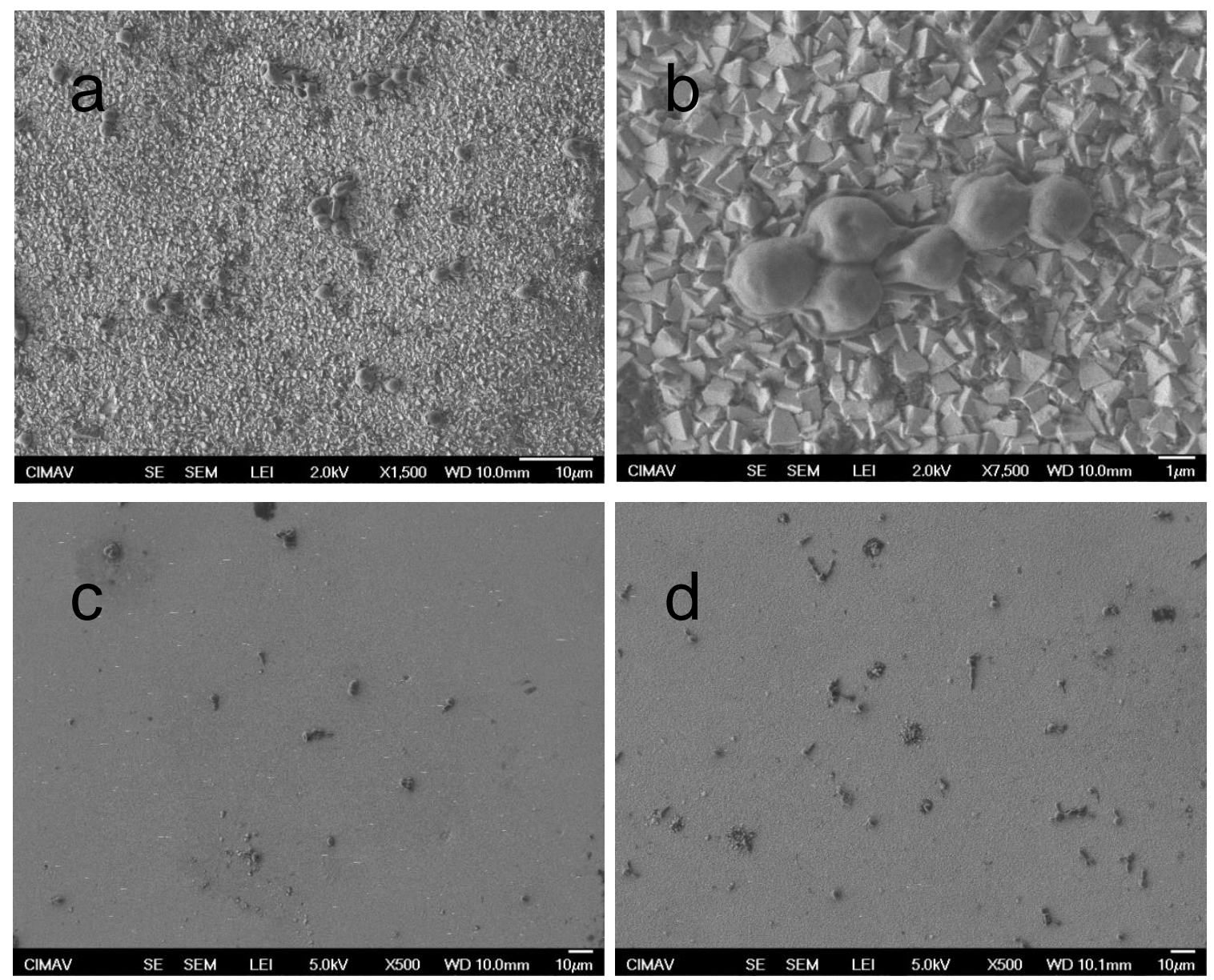

Figure 2. a) Micrograph at 1,500 augments of C. albicans adhered in the $\mathrm{SnO} 2$ thin film. b) C. albicans at 7,500 augments on a $\mathrm{SnO} 2$ thin film. c-d) Comparison of number of cell yeast of C. albicans: c) before UV exposure, d) after 15 minutes of UV exposure.

\section{References}

[1] Singh, S. B., Young, K., \& Silver, L. L. (2017). What is an "ideal” antibiotic? Discovery challenges and path forward. Biochemical Pharmacology, 63-73.

[2] Edwards, M., Hamilton, R., Nicola, O., Fitzgibbon, S., \& Samarasekera, R. (2019). Antibiotic Resistance: Modeling the Impact on Mortality and Morbidity. London: Institute and Faculty of Actuaries. [3] Fakhri, A., Behrouz, S., \& Pourmand, M. (2014). Synthesis, photocatalytic and antimicrobial properties of $\mathrm{SnO} 2, \mathrm{SnS} 2$ and $\mathrm{SnO} 2 / \mathrm{SnS} 2$ nanostructure. Journal of Photochemistry and Photobiology B: Biology, (149)45-50.

[4] Fuentes, N., O'Hara, L. M., \& Harris, A. D. (2019). Transmission pathways of multidrug-resistant organisms in the hospital setting: a scoping review. Infection Control \& Hospital Epidemiology, 1-10.

[5] Javadia, E., Ghaffaria,M., Bahlakehb, G., Taheric, P. (2019). Photocatalytic, corrosion protection and adhesion properties of acrylic nanocomposite coating containing silane treated nano zinc oxide: A combined experimental and simulation study. Progress in Organic Coatings (135)496-509.

[6] Mohri,N., Kerschbaumer H. Link, T., Andre, R., Panthöfer, M., Ksenofontov, V., Tremel W. (2019). Self-Organized Arrays of SnO2 Microplates with Photocatalyticand Antimicrobial Properties. Eur. J. Inorg. Chem. 3171-3179 
[7] Pandiyan, R., Mahalingam, S., \& Ahn, Y.-H. (2019). Antibacterial and photocatalytic activity of hydrothermally synthesized $\mathrm{SnO} 2$ doped $\mathrm{GO}$ and $\mathrm{CNT}$ under visible light irradiation. Journal of Photochemistry \& Photobiology, B: Biology, (191)18-25. 